\title{
Adaptive Gearbox Selection Method to Improve the Performance of Industrial Robotic Arms
}

DOI 10.1515/jisys-2016-0225

Received October 1, 2016; previously published online April 19, 2017.

\begin{abstract}
Robotic arms play a major role in today's environment. Many robotic arms are used in industries, factories, and our surrounding environments. Many types of robotic arms are used for their particular purpose. Gearbox selection is very important in increasing the performance of robotic arms. Therefore, it is necessary to select the gearbox to improve the performance of robotic arms for everyday use. Thus, an efficient method is proposed to improve the performance of robotic arms for industries. This efficient method adaptively controls the gearbox to increase the performance of industrial robotic arms. The cuckoo search algorithm is an efficient method proposed to increase the performance of industrial robotic arms. By this method, we can improve the performance of industrial robotic arms and make use of these robotic arms in the modern world. The technique has been implemented in the working platform of MATLAB, and the results have been analyzed.
\end{abstract}

Keywords: Robotic arms, gearbox, cuckoo search algorithm.

\section{Introduction}

Robots are simple or complex machines that can execute a wide range of jobs done by humans with the help of a set of sensors and effectors. The sensors help the robots in recognizing their surrounding environment, while the effectors allow them to act upon physically. The robots can be classified into three major types, namely manipulators, mobile robots, and humanoid robots [12]. Robots find massive application in industries, to deliver products with better quality and safety while having an increased control over the cost and processing time. The model of a robot is developed based on the inertia, mass, and center of mass of each link in it [1].

Robotics depends on two technologies that make it possible. They are the tele-manipulators and the ability to numerically control the machines. Tele-manipulators are machines that can be controlled from a distance with the help of an arm and a gripper that make movements as directed by the human controller through a control device, whereas numeric control makes use of a coordinate system to have an accurate control over the machines [12].

\subsection{Types of Robot}

\subsubsection{Mobile Robots}

The mobile robots are a kind of robots that utilize a special group of effectors like wheels, tracks, and legs for their motion. The differential drive has two wheels, one on each side acting separately and not influencing

*Corresponding author: Mohammad Athar Hayat, Manoharbhai Patel Institute of Engineering \& Technology, Gondia, Maharashtra, India, e-mail: mohammadatharhayat0581@gmail.com

Ankush A. Mankar: V.M Institute of Engineering and Technology, Maharashtra, India 
one another. The robot travels in a straight line if the two wheels move at the same speed and in the same direction. The robot turns on the spot if it moves in the reverse direction. The mobile robot was basically developed for the intention of automating transportation in production processes and autonomous transport systems. However, later on, nature-inspired robots like insectoid robots that have many legs and autonomous robots that can be used under water have been constructed.

\subsubsection{Hardworking Robots}

The robots have been used as substitutes to human workers where the risk of danger is high and the working environment proves to be harder, that some kind of automation is necessary. This may include the assembly line work in the automobile industry or the harvesting machines used in the agricultural field. In addition to assembly robots, other prevailing robots are the melon harvester robot, the ore transport robot for mines, the robot that removes paint from huge ships, and the robot that can create highly accurate sewer maps. Robots prove to be faster with lower expense and increased accuracy than humans if they are provided with an appropriate working environment.

\subsubsection{Transporters}

Despite the changes that they require in the working area to carry out their tasks, most autonomous transport robots are currently being used in practice. Now, constructing a robot that can make movements using natural landmarks is never impossible. Some of the recently existing transporters include container transporters that are used to load and unload cargo ships, medication and food transport systems in hospitals, and autonomous helicopters that are used for the distribution of goods in remote regions.

\subsubsection{Insensible Steel Giants}

The robots are capable of working in an unsafe, poisonous, or nuclear environment as it is merely a machine that can be easily safeguarded and replaceable. They help in cleaning up the wastes that result from nuclear processes, ammunition, and mines. One such robot is the Chernobyl robot that removes nuclear waste. In addition to waste removal, the robots can be sent to other planets like Mars or into the bottom of the ocean to search for the craters of active volcanoes or sunken ships, as they remain unaffected in hazardous environments.

\subsubsection{Servants and Toys}

Though robots are not much common in usage, they have been widely used as toys or household helpers [12]. They attract children in the form of toys, such as Sony Aibo, and help old people in having a well-protected life.

\subsubsection{Industrial Robots}

Industrial robots have had a remarkable usage in the manufacturing sector for $>30$ years, in performing tasks like stacking, casting, painting, sorting, welding, component soldering, and so on. The industrial robots have a long heritage in the manufacturing industry by doing tasks that are difficult for humans, in a continuous manner with more accuracy and speed. They work in relatively static environments and in large numbers. 
The oil and gas industry recommend the usage of industrial robots to have increased protection and efficiency with less environmental impact. Remotely controlled industrial robots can be used to replace humans when maintenance, inspection, and repairs are to be undertaken in a danger zone [7].

\subsection{Gearbox}

The speed from the low rotating rotor to the high rotating generator can be varied by means of a gearbox [4]. Robotic systems that offer high torques at the end effectors often have high reduction gears in their transmissions that cause gear-specific friction components, like position-dependent friction, to occur. The positiondependent friction is the force that comes into action when a pair of teeth meet, and a periodic waveform is generated with the frequency with which the two teeth match.

The function of gears can be examined with the following parameters:

(a) Direction of turning;

(b) Relative speed;

(c) Number of revolutions;

(d) Mechanical advantages pertaining to the principles of power and speed [2].

While power and load in gear trains are transmitted along the line of action direction, friction travels orthogonal to it. This friction results from the imperfect rolling and sliding of teeth. Hence, the inefficiency in gearboxes is due to the meshing friction. Small shaft displacements along the line-of-action direction cause torque oscillations, which, in turn, are caused by the relative reduced stiffness of shaft support ball bearings. This is called position-dependent friction. The meshing friction force in gear teeth is transmitted in the off line of the action direction. The friction coefficient between gear teeth relies on the lubricant properties, and it is inversely proportional to the relative sliding velocity between gear teeth [11].

\subsection{Types of Gears}

The type of gear heavily decides the amplitude of oscillation caused by meshing friction [5]. The meshing friction is small in case of spur or helical gears where the operating principle is rolling between teeth. However, in case of worm gears that operate with almost pure sliding friction, the friction decreases with increase in speed and availability of sufficient lubricant film that prevents contact between asperities.

The various gear types are as follows:

- External and internal gears;

- Spur gear;

- Helical gears;

- Skew gears;

- Double helical gears;

- Bevel gears;

- Hypoid gears;

- Crown gears;

- Worm gear;

- Non-circular gears;

- Rack and pinion gearing;

- Epicyclic gearing;

- Sun and planet gearing;

- Harmonic drive gearing;

- Cage gear. 


\subsection{Common Gearbox Parameters}

The common gearbox parameters are described below.

\subsubsection{Viscous Friction Coefficient}

When two surfaces are in relative motion, the viscous friction between them is based on the dimensional parameters such as area and clearance between the two surfaces as well as the fluid properties such as fluid specific gravity and viscosity. The viscous friction is found to be inversely proportional to the clearance between the two meeting surfaces [9].

\subsubsection{Coulomb Friction Coefficient}

When two dry surfaces are in contact with each other, the friction force that exists between them is called the Coulomb friction. The Coulomb friction coefficient is a unitless number that is known for many simple, pure materials, and is found to be a static force that is slightly higher than the motive force when two resting materials are in contact. The coefficient of friction for wood against concrete is 0.62 ; for polystyrene against steel, it is 0.3-0.35; and for steel against Teflon, it is 0.04. Multiplying the normal force with the coefficient of friction will allow the calculation of the force required to overcome static friction or friction force. The normal force is the mass of the material times the gravitational pull, and it gets added up in vector calculations if the two surfaces move up or down an incline with the gravitational pull acting toward or away from it [3].

\subsubsection{Striebeck Friction Coefficient}

It is given by the variation of friction between two liquid lubricated surfaces as a function of a dimensionless lubrication parameter $\eta N / P$, where $\eta$ is the dynamic viscosity, $N$ the speed (e.g. revolutions per minute of a bearing), and $P$ the load projected onto the geometrical surface [14].

\subsubsection{Friction Smoothness Coefficient}

Friction is defined as the resistance that is encountered by an object in moving over another (Oxford English Dictionary). Dragging an object over glass seems to be easier than over a sandpaper, as the sandpaper exerts more frictional resistance. A surface is termed "smooth" if it does not exert any frictional force and is termed "rough" if some kind of frictional resistance exists [13].

\subsubsection{Total Moment of Inertia}

By classical mechanics, moment of inertia, also known as mass moment of inertia, rotational inertia, polar moment of inertia of mass, or the angular mass (SI unit: $\mathrm{kg} \cdot \mathrm{m}^{2}$ ), is defined as a measure of an object's resistance to changes to its rotation. It is the inertia of a rotating body with respect to its rotation. The role of moment of inertia in rotational dynamics can be viewed as the role of mass in linear dynamics, describing the relationship between angular momentum and angular velocity, torque and angular acceleration, and several other quantities. The symbol $I$ or $J$ often indicates the moment of inertia or polar moment of inertia [10]. 


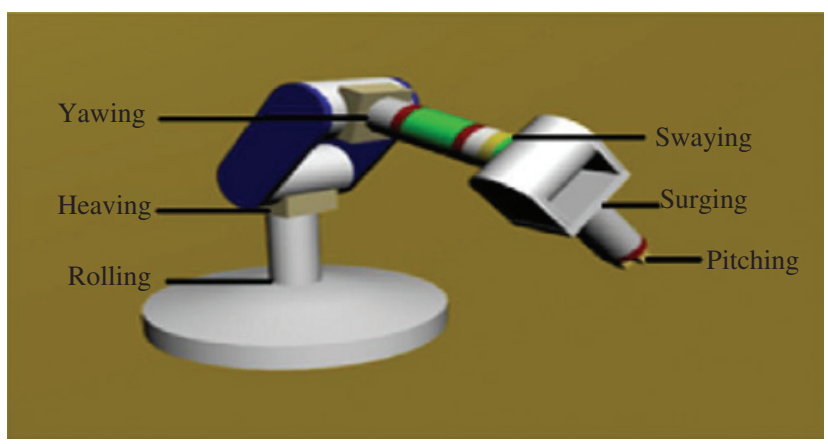

Figure 1: An Articulated Robot with Six dof in a Kinematic Chain.

\section{Related Works}

Hamon et al. [6] proposed the dynamic identification of robots with a load-dependent joint friction model. They proposed a new inverse dynamic identification model for $n$ degrees of freedom (dof) serial robot, where the dry friction force is a linear function of both the dynamic and the external forces (Figure 1). This groups all the joint data collected while the robot is tracking planned trajectories with different payloads to get a global least squares estimation, in one step, of inertial and new friction parameters. They also carried out an experimental validation with a 1 dof prismatic joint composed of a Star high precision ball screw drive positioning unit, which allowed large and easy variations of the inertial and gravity forces.

Wernholt and Moberg [16] proposed frequency-domain gray-box identification of industrial robots. They found the unknown parameters in elastic dynamic models of industrial robots. These parameters were identified in the frequency domain, using estimates of the non-parametric frequency response function (FRF) in different robot configurations/positions. The non-linear parametric robot model was linearized in the same positions, and the optimal parameters were obtained by minimizing the discrepancy between the non-parametric FRFs and the parametric FRFs (the FRFs of the linearized parametric robot model).

Wernholt and Gunnarsson [15] proposed a non-linear identification of a physically parameterized robot model. Their parameters were identified only using motor measurements. In their first step, they identified rigid body dynamics and friction using a separable least squares method, where a friction model described the Striebeck effect was used. In their second step, initial values for flexibilities were obtained using the inverse eigenvalue theory. Finally, in their last step, the remaining parameters of a non-linear physically parameterized model were identified directly in the time domain. Their procedure was exemplified by using real data from an experimental industrial robot.

Wernholt and Moberg [17] proposed non-linear gray-box identification using local models applied to industrial robots. They linearized the non-linear gray-box model in the same operating points, resulting in parametric FRFs. They obtained the optimal parameters finally by minimizing the discrepancy between the non-parametric and parametric FRFs. They also illustrated their procedure by estimating elasticity parameters in a six-ax industrial robot. They compared different parameter estimators, and their experimental results showed the usefulness of their proposed identification procedure.

\section{Calculation of Degree of Freedom}

\subsection{Six dof}

\section{Translation:}

1. Moving up and down (heaving);

2. Moving left and right (swaying);

3. Moving forward and backward (surging); 
Rotation

1. Tilting forward and backward (pitching);

2. Turning left and right (yawing);

3. Tilting side to side (rolling).

The dof, or mobility, of a kinematic chain is the number of parameters that define the configuration of the chain. A system of $n$ rigid bodies moving in space has $6 n$ dof measured relative to a fixed frame. This frame is included in the count of bodies, so that mobility does not depend on the link that forms the fixed frame. This means the dof of this system is $M=6(N-1)$, where $N=n+1$ is the number of moving bodies plus the fixed body.

Joints that connect bodies impose constraints. Specifically, hinges and sliders each impose five constraints and therefore remove 5 dof. It is convenient to define the number of constraints $c$ that a joint imposes in terms of the joint's freedom $f$, where $c=6-f$. In the case of a hinge or slider, which has 1 dof joint, $f=1$ and therefore $c=6-1=5$.

The result is that the mobility of a kinematic chain formed from $n$ moving links and $j$ joints each with freedom $f_{i}, i=1, \ldots, j$, is given by

$$
M=6 n-\sum_{i=1}^{j}\left(6-f_{i}\right)=6(N-1-j)+\sum_{i=1}^{j} f_{i} .
$$

\subsubsection{Spatial Rotational Transformation}

We can describe a spatial rotation operator for the rotational transformation of a point about an unit axis $\mathbf{u}$ passing through the origin of the coordinate system. Suppose the rotational angle of the point about $u$ is $\theta$, the rotation operator will be expressed by

$$
R_{\theta, u}=\left[\begin{array}{cccc}
u_{x}^{2} v \theta+c \theta & u_{x} u_{y} v \theta-u_{z} s \theta & u_{x} u_{z} v \theta+u_{y} s \theta & 0 \\
u_{x} u_{y} v \theta+u_{z} s \theta & u_{y}^{2} v \theta+c \theta & u_{y} u_{z} v \theta-u_{x} s \theta & 0 \\
u_{x} u_{z} v \theta-u_{y} s \theta & u_{y} u_{z} v \theta+u_{x} s \theta & u_{z}^{2} v \theta+c \theta & 0 \\
0 & 0 & 0 & 1
\end{array}\right],
$$

where $u_{x}, u_{y}, u_{z}$ are the othographical projection of the unit axis $\mathbf{u}$ on $x, y$, and $z$ axes, respectively:

$$
\begin{aligned}
& s \theta=\sin \theta, \\
& c \theta=\cos \theta, \\
& v \theta=1-\cos \theta .
\end{aligned}
$$

\subsubsection{Spatial Translational Transformation}

Suppose that a point $P$ on a rigid body goes through a translation describing a straight path from $P_{1}$ to $P_{2}$ with a change of coordinates of $(\Delta x, \Delta y, \Delta z)$; we can describe this motion with a translation operator $T$ :

$$
T=\left[\begin{array}{cccc}
1 & 0 & 0 & \Delta x \\
0 & 1 & 0 & \Delta y \\
0 & 0 & 1 & \Delta z \\
0 & 0 & 0 & 1
\end{array}\right]
$$

\section{Cuckoo Search Algorithm-Based Optimized Parameter Value}

Based on the manner of life of a cuckoo bird, one of the newly grown bio-inspired algorithms is the cuckoo search (CS). Cuckoos utilize a forceful strategy of reproduction in which they occupy the female hew nests of 
other birds to put down their fertilized eggs. Occasionally, the egg of cuckoo in the nest is revealed, and the hacked birds throw away or abandon the nest and place their own offspring somewhere else. Based on the subsequent three idealized rules, the CS was proposed by as follows:

- Every cuckoo lays one egg at a time, and deposits it in a erratically chosen nest.

- The top nests with a high class of eggs (solutions) will be taken over to the next generations.

- The number of existing host nests is fixed, and a host can find out an alien egg with a possibility $p a \in[0,1]$. In this case, to build an entirely new nest in a new place, the host bird can either throw the egg away or discard the nest [8].

An essential advantage of this algorithm is its plainness. There is basically only a single parameter in CS (apart from the population size) comparing with other population- or agent-based metaheuristic algorithms such as particle swarm optimization and harmony search. The CS pseudocode of the CS is set in the following.

\subsection{Pseudocode of the CS Algorithm}

\begin{tabular}{|l|}
\hline begin \\
Objective function $f(z), z=\left(z_{1}, z_{2}, \ldots . . z_{d}\right)$ \\
Generate initial population of \\
$n$ host nests \\
while ( $t<$ MaxGeneration) or \\
criterion) \\
Get a cuckoo randomly by Levy flights \\
evaluate its quality/fitness $F_{i}$ \\
Choose a nest among $n$ (say, $j)$ randomly \\
if $\left(F_{i}>F_{j}\right)$, \\
replace $j$ by the new solution; \\
end if \\
A fraction ( pa) of worse nests \\
are abandoned and new ones are built; \\
Keep the best solutions \\
(or nests with quality solutions); \\
generation \\
end while solutions and find the current best
\end{tabular}


From the above pseudocode, select the number of population to be applied. To compute the fitness, all the obtained population and the corresponding parameter values are taken from Eq. (1). Here, velocity maximization is the fitness function, which is shown below.

Here, the objective function

$$
\begin{gathered}
f(z)=y_{3}(z), \\
\mathrm{d} / \mathrm{d} z y_{1}(z)=y_{3}(z)-y_{4}(z), \\
\mathrm{d} / \mathrm{d} z y_{2}(z)=y_{4}(z)-y_{5}(z), \\
\mathrm{d} / \mathrm{d} z y_{3}(z)=1 /\left(I^{\star} m_{-} i\right)\left(-S P_{t}\left(y_{1}(z)\right)-G_{d}\left(y_{3}(z)-y_{4}(z)\right)-F R_{t}\left(y_{3}(z)\right)+u(z)\right), \\
\mathrm{d} / \mathrm{d} z y_{4}(z)=1 /\left(I^{\star} g_{-} i\right)\left(S P_{t}\left(y_{1}(z)\right)+G_{d}\left(y_{3}(z)-y_{4}(z)\right)-K_{s} y_{2}(z)-A_{d}\left(y_{4}(z)-y_{5}(z)\right)\right), \\
\mathrm{d} / \mathrm{d} z y_{5}(z)=1 /\left(I\left(1-m_{-} i-g_{-} i\right)\left(K_{s} y_{2}(z)+A_{d}\left(y_{4}(z)-y_{5}(z)\right)\right) .\right.
\end{gathered}
$$

Here,

$y_{1}(z)=$ angular position difference between the motor and the gearbox

$y_{2}(z)=$ angular position difference between the gearbox and the arm

$y_{3}(z)=$ angular velocity of motor

$y_{4}(z)=$ angular velocity of gearbox

$y_{5}(z)=$ angular velocity of robot arm

$I=$ total moment of inertia

$M_{i}=$ moment of inertia of motor

$G_{i}=$ moment of inertia of gearbox

$A_{i}=$ moment of inertia of arm

$m \_i=$ motor moment of inertia scale factor

$g_{\_} i=$ gearbox moment of inertia scale factor

$u(z)=$ applied motor torque

$A_{d}=$ damping parameters of arm

$G_{d}=$ damping parameters of gearbox

$K_{s}=$ stiffness of the second spring

$$
F R_{t}\left(y_{3}(z)\right)=V_{c}\left(y_{3}(z)\right)+\left(C_{c}+S b_{c} \operatorname{sech}\left(\sigma y_{3}(z)\right)\right) \tanh \left(\tau y_{3}(z)\right)
$$

$V_{c}=$ viscous friction coefficient

$C_{c}=$ Coulomb friction coefficient

$S b_{c}=$ Striebeck friction coefficient

$\sigma=$ Striebeck smoothness coefficient

$\tau=$ friction smoothness coefficient

$F R_{t}=$ friction torque

$$
S P_{t}\left(y_{1}(z)\right)=K_{g 1} y_{1}(z)+K_{g 3} y_{1}(z)^{3}
$$

$K_{g 1}=$ gearbox stiffness parameter 1

$K_{g 2}=$ gearbox stiffness parameter 3

$S P_{t}=$ spring torque 
Algorithm

Step 1: Initialize all the input of the system.

Step 2: Find the fitness of the system using following equation:

$$
\text { Fitness }=\max [f(z)] .
$$

Step 3: Determine the better fitness and generate the new solution using the following equation:

$$
X_{i}^{t+1}=X_{i}^{t}+\alpha \oplus \operatorname{Levy}(\lambda),
$$

where $a>0$ is the step size, which should be related to the scale of the problem of interest; the product $\oplus$ means entry-wise multiplications. In this research work, we consider a Levy flight in which the step lengths are distributed according to the following probability distribution:

$$
\operatorname{Levy}(\lambda)=t^{-\lambda}, 1<\lambda \leq 3 \text {. }
$$

Step 4: Find the fitness probability rate using $p a \in[0,1]$, whereas the best solution can be determined by the maximum angular velocity.

Step 5: Terminate the process.

\section{Results and Discussion}

The adaptive gearbox selection method is shown in the result given below with the angular velocity of motor with respect to time. By analyzing these results, we can conclude that the adaptive gearbox selection method improves the performance of industrial robotic arms.

\subsection{Discussion}

From Figures 2-5, we can see the fitness of angular velocity of motor with respect to time in robotic arms. The fitness of the first and second diagrams gives the values of 87.55 and 88.79, which are high, whereas the fitness value of the third diagram is $64.46 \%$. In the fourth diagram, the fitness value is very high at $99.92 \%$. Thus, from the figure, it is clear that our proposed method shows good efficiency. Hence, the model is able to select the adaptive gearbox for the good performance of industrial robotic arms. Therefore, we can say that our proposed method is very useful in the selection of gearbox for the better performance of industrial robotic arms. The model will be more precise when compared to other conceptual models.

\section{Conclusions}

The proposed technique was robust and used to predict the optimized value from the generated population. In this paper, the proposed method using the CS algorithm for the industrial robotic arm process was illustrated in detail. The results presented in this paper showed that our technique is more effective in selecting the gearbox in industrial robotic arms. We can clearly see from the figures that the fitness values are very high. Therefore, in conclusion, we can clearly say that our proposed method is very efficient in the adaptive gearbox selection method for the performance of industrial robotic arms. Also, the proposed method helps in increasing the efficiency of the gearbox selection method. Thus, we can say that, in the future, our proposed method will be very helpful in selecting the gearbox for the performance of industrial robotic arms, and that it 


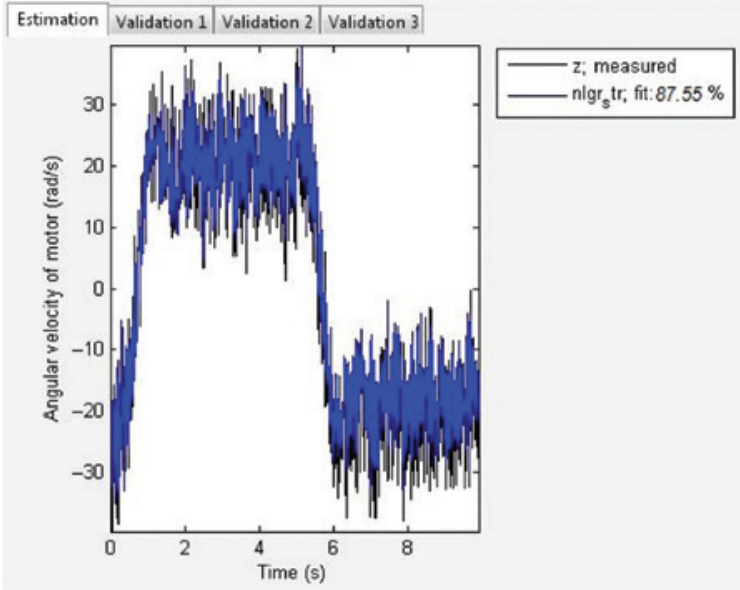

Figure 2: Fitness Value (1) of Motor.

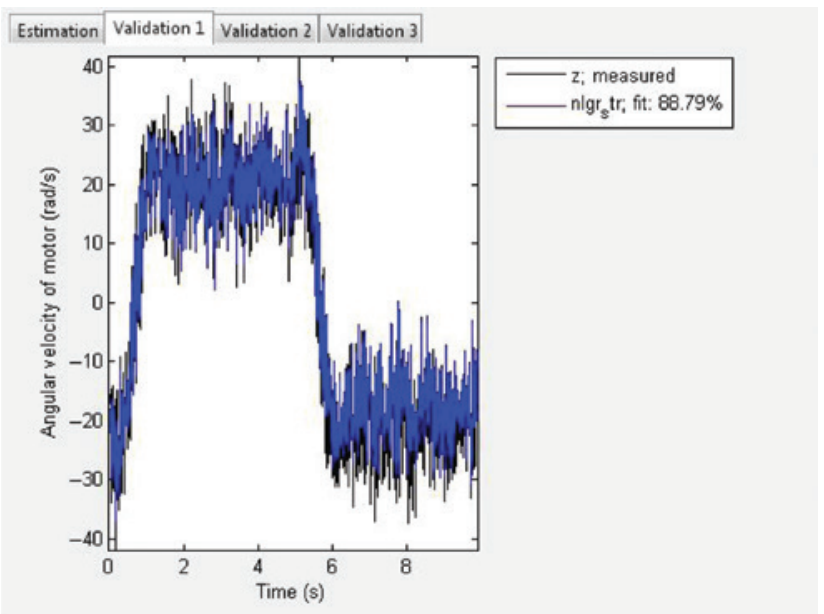

Figure 3: Fitness Value (2) of Motor.

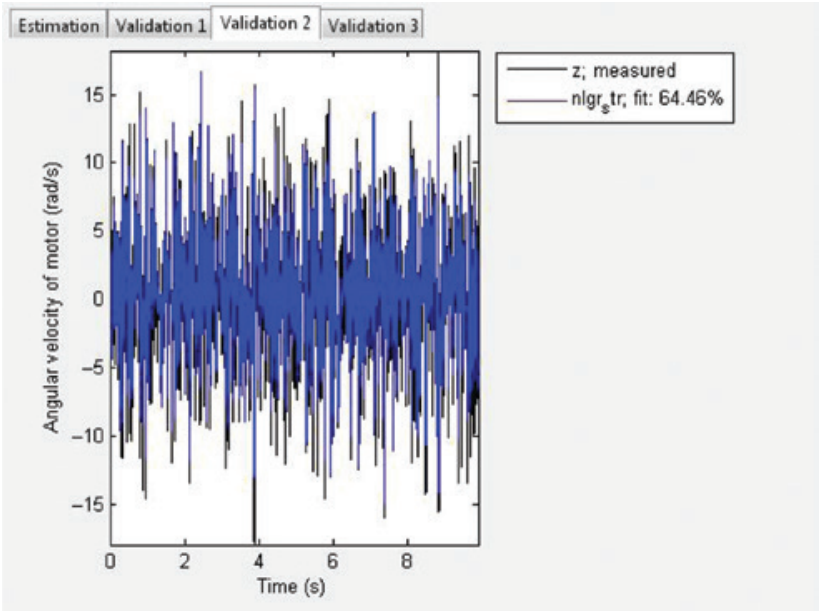

Figure 4: Fitness Value (3) of Motor. 


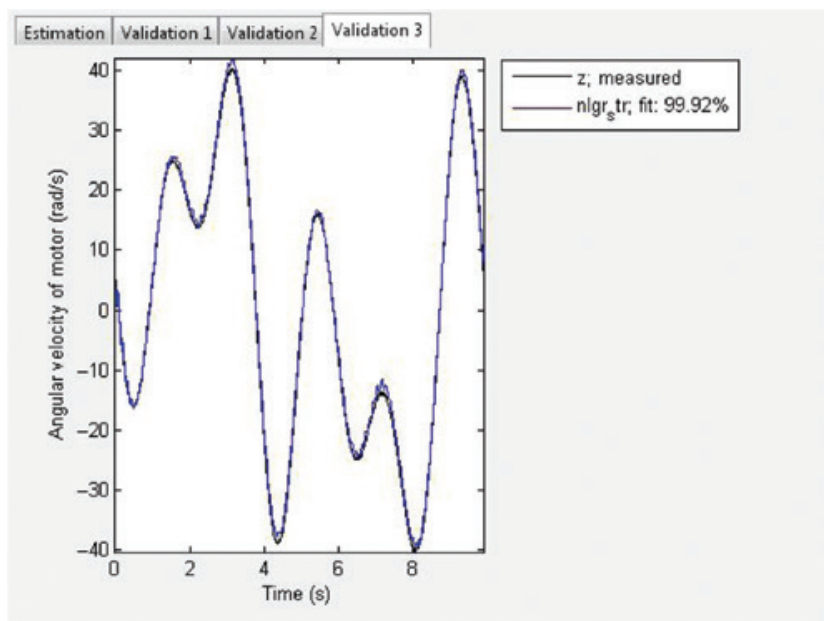

Figure 5: Fitness Value (4) of Motor.

is very useful for the improvement of industrial robots. Therefore, the performance of industrial robotic arms can be made very effective by using our proposed method.

\section{Bibliography}

[1] B. Bona and A. Curatella, Identification of industrial robot parameters for advanced model-based controllers design, In: Proceedings of the 2005 IEEE International Conference on Robotics and Automation (ICRA), pp. 1681-1686, Barcelona, Spain, 2005.

[2] J. M. Chambers, M. Carbonaro and H. Murray, Developing conceptual understanding of mechanical advantage through the use of Lego robotic technology, Australas. J. Educ. Technol. 24 (2008), 387-401.

[3] M. R. Cutkosky and R. D. Howe, Practical force-motion models for sliding manipulation, Int. J. Robot. Res. 15 (1996), $557-572$.

[4] S. Gade, R. Schlombs, C. Hundeck and C. Fenselau, Operational modal analysis on a wind turbine gearbox, In: Conference \& Exposition on Structural Dynamics, 2009.

[5] E. Garcia, P. G. de Santos and C. C. de Wit, Velocity dependence in the cyclic friction arising with gears, Int. J. Robot. Res. 21 (2002).

[6] P. Hamon, M. Gautier, P. Garrec and A. Janot, Dynamic identification of robot with a load-dependent joint friction model, In: Proceedings IEEE Conference on Robotics Automation and Mechatronics (RAM), pp. 129-135, 2010.

[7] C. Heyer, Human-robot interaction and future industrial robotics applications, In: IEEE/RSJ International Conference on Intelligent Robots and Systems (IROS), 2010.

[8] S. E. Hodges and R. J. Richards, Look and learn: towards cheap, flexible robots, In: Proceedings of the International Conference on Recent Advances in Mechatronics ICRAM, 1995.

[9] M. K. B. Khalil, Estimated versus calculated viscous friction coefficient in spool valve modeling, In: Technical Conference on IFPE, 2008.

[10] C. Leng, Q. Cao and Y. Huang, A motion planning method for omni-directional mobile robot based on the anisotropic characteristics, Int. J. Adv. Robot. Syst. 5 (2008), 45.

[11] C. Makkar, W. E. Dixon, W. G. Sawyer and G. Hu, A new continuously differentiable friction model for control systems design, In: Proceedings IEEE/ASME International Conference on Advanced Intelligent Mechatronics, pp. 600-605, September 26, 2005.

[12] T. Niemueller and S. Widyadharma, Artificial intelligence - an introduction to robotics, Al-Robotics, 8 (2003), 1-14.

[13] B. N. J. Persson, Theory of rubber friction and contact mechanics, J. Chem. Phys. 115 (2001), 3840-3861.

[14] K. S. Reddy, P. V. K. Perumal, B. Durgaprasad and M. A. Murtaza, Optimizing the performance evaluation of robotic arms with the aid of particle swarm optimization, Int. J. Adv. Comput. Sci. Appl. 3 (2012).

[15] E. Wernholt and S. Gunnarsson, Nonlinear identification of a physically parameterized robot model, In: 14th IFAC Symposium on System Identification, 2006.

[16] E. Wernholt and S. Moberg, Frequency-domain gray-box identification of industrial robots, In: 17th IFAC World Congress, pp. 15372-15380, 2008.

[17] E. Wernholt and S. Moberg, Nonlinear gray-box identification using local models applied to industrial robots", Automatica 47 (2011), 650-660. 